\title{
TOSHIO NAKAGAWA
}

\section{AMRIT L. GOEL}

\section{SHUNJI OSAKI}

\section{Stoghastig behavior of an intermittently used system}

Revue française d'automatique, d'informatique et de recherche opérationnelle. Recherche opérationnelle, tome 9, $\mathrm{n}^{\circ}$ V2 (1975), p. 101-112.

<http://www.numdam.org/item?id=RO_1975_9_2_101_0>

(C) AFCET, 1975, tous droits réservés.

L'accès aux archives de la revue « Revue française d'automatique, d'informatique et de recherche opérationnelle. Recherche opérationnelle » implique l'accord avec les conditions générales d'utilisation (http://www.numdam.org/ legal.php). Toute utilisation commerciale ou impression systématique est constitutive d'une infraction pénale. Toute copie ou impression de ce fichier doit contenir la présente mention de copyright.

\section{Numdam}

Article numérisé dans le cadre du programme

Numérisation de documents anciens mathématiques

http://www.numdam.org/ 


\title{
STOGHASTIC BEHAVIOR \\ OF AN INTERIMITTENTLY USED SYSTEM (*)
}

\author{
by Toshio Nakagawa $\left({ }^{1}\right)$, Amrit L. Goel $\left({ }^{2}\right)$ \\ and Shunji OSAKI $\left({ }^{3}\right)$
}

\begin{abstract}
This paper discusses the stochastic behavior of an intermittently used system. Of interest are (i) the distribution of the disappointment time, (ii) the expected number of disappointments during a finite interval, and (iii) the pointwise availability. This paper derives the above three measures applying a Markov renewal process. A few examples are also presented.
\end{abstract}

\section{INTRODUCTION}

In general, a system can be classified into one of two types according to whether it is always used or it is intermittently used. In this paper we are interested in an intermittently used system. That is, a system which alternatively operative or inoperative, but is used intermittently. Many examples of such a system occur in traffic and scheduling studies as well as in reliability studies.

Gaver [4] introduced the so-called « disappointment time », the time to system failure during a usage period or to occurrence of a need during a system inoperative period, whichever occurs first. He derived the LaplaceStieltjes $(L S)$ transform and the mean of the disappointment time distribution. Osaki [5] discussed the disappointment time for an intermittently used system introducing preventive maintenance policies. Srinivasan [7] studied the disappointment time for a two-unit standby redundant system which is used intermittently. Earlier contributions have been mainly made to the behavior of the first disappointment time. Another useful measure in reliability theory is the availability.

(*) Reçu juillet 1974.

(1) Department of Mathematics, Meijo University, Nagoya, Japan.

(2) Department of Industrial Engineering and Operations Research, Syracuse University, Syracuse, New York.

(3) Department of Industrial Engineering, Hiroshima University, Hiroshima, Japan.

Revue Française d'Automatique, Informatique et Recherche Opérationnelle, $\mathrm{n}^{\circ}$ juin $1975, \mathrm{~V}-2$ 
In this paper we discuss the availability of an intermittently used system, i.e. the stochastic behavior of the system including the behavior after the disappointment time. Consider a one-unit system subject to failure and repair, where an operative period means the time to failure and an inoperative period means the repair time duration. A need occurs randomly and the holding time for each need is also random, which is independent of the behavior of the unit. The disappointment occurs (a) when the unit fails during a usage period or (b) when the unit is needed during its repair period, whichever occurs first. We further assume that if the unit fails during a usage period, it is again used immediately after completion of the repair. We also assume that if the unit is needed during its repair period, it begins to be used immediatly after completion of the repair. These assumptions seem to be plausible in practice. Suppose that the unit begins to be operative and a need does not occur at time 0 . Then we are interested in the following :

(i) The distribution of the disappointment time.

(ii) The expected number of disappointments during a finite interval.

(iii) The pointwise unavailability, i.e., the probability that the system is inoperative during a usage period, or a need occurs during a system inoperative period.

We derive the above measures using mass functions of a Markov renewal process and renewal-type equations. Our method mentioned in this paper has a wide applicability in operations research as well as in reliability theory. We also discuss a similar model in which the behavior of a need obeys an equilibrium alternating renewal process. A few examples are also presented.

\section{MODEL AND ANALYSIS}

Consider a one-unit system subject to failure and repair. The time to failure has an arbitrary distribution $F(t)$ and the repair time of the failed unit has an arbitrary distribution $G(t)$. Both the mean failure and repair times are assumed to be finite and are denoted by $1 / \alpha$ and $1 / \beta$, respectively. We assume that each switchover is perfect and each switchover time is instantaneous. We also assume that the unit is as good as new upon repair.

Let us talk about the behavior of the needs for the unit. We assume that occurrence and the holding time of a need are both random with parameters $\lambda$ and $\mu$, respectively. That is, a need occurs with the exponential distribution $1-\exp (-\lambda t)$ and the holding time of a need has the exponential distribution $1-\exp (-\mu t)$. Define state 1 when the unit is needed and state 0 when the unit is not needed. Then the behavior of the needs assumes 0 and 1 alternatively. Let $H_{i j}(t)$ denote the probability that the unit is in state $j$ at time $t$, given that it was in state $i$ at time $0(i, j=0,1)$. From Barlow and Hunter [1], we have 


$$
\begin{aligned}
& H_{00}(t)=\frac{\mu}{\lambda+\mu}+\frac{\lambda}{\lambda+\mu} \exp [-(\lambda+\mu) t], \\
& H_{01}(t)=\frac{\lambda}{\lambda+\mu}-\frac{\lambda}{\lambda+\mu} \exp [-(\lambda+\mu) t], \\
& H_{10}(t)=\frac{\mu}{\lambda+\mu}-\frac{\mu}{\lambda+\mu} \exp [-(\lambda+\mu) t], \\
& H_{11}(t)=\frac{\lambda}{\lambda+\mu}+\frac{\mu}{\lambda+\mu} \exp [-(\lambda+\mu) t] .
\end{aligned}
$$

It is evident that the behavior of an intermittently used system can be described by the interaction between two alternating renewal processes in which the regeneration points are the time instants at which failure occurs and at which the repair is completed. It is also noted that the behavior of the needs has the memoryless properties because of the exponential assumptions. Therefore, we define the following regeneration points :

State $0:$ The unit begins to be operative while it is not needed.

State 1 : The unit begins to be repaired while it is not needed.

State 2 : The unit begins to be operative while it is needed.

State 3 : The unit begins to be repaired while it is needed.

Note that the disappointment time happens either when the process makes a transition into state 3 , or when a need occurs while the unit is under repair (from state 2).

The states defined above are regarded as those of a Markov renewal process. From the theory of Markov renewal processes [6], the following mass functions of the Markov renewal process are derived :

$$
\begin{aligned}
& Q_{01}(t)=\int_{0}^{t} H_{00}(t) \mathrm{d} F(t) \\
& Q_{03}(t)=\int_{0}^{t} H_{01}(t) \mathrm{d} F(t), \\
& Q_{10}(t)=\int_{0}^{t} \mathrm{e}^{-\lambda t} \mathrm{~d} G(t), \\
& Q_{12}(t)=\int_{0}^{t}\left(1-\mathrm{e}^{-\lambda t}\right) \mathrm{d} G(t), \\
& Q_{21}(t)=\int_{0}^{t} H_{10}(t) \mathrm{d} F(t), \\
& Q_{23}(t)=\int_{0}^{t} H_{11}(t) \mathrm{d} F(t), \\
& Q_{32}(t)=G(t),
\end{aligned}
$$

$n^{\circ}$ juin 1975, V-2 
and $Q_{i j}(t) \equiv 0$ for otherwise. The mass function denotes the probability that after making a transition into state $i$, the process next makes a transition into state $j$, in an amount of time less than or equal to $t$, in a Markov renewal process (see Pyke [6]). Let $q_{i j}(s)$ denote the $L S$ transform of the mass function $Q_{i j}(t)$. Then the $L S$ transforms $q_{i j}(s)$ in (5)-(11) are

$$
\begin{aligned}
& q_{01}(s)=\frac{\mu}{\lambda+\mu} f(s)+\frac{\lambda}{\lambda+\mu} f(s+\lambda+\mu), \\
& q_{03}(s)=\frac{\lambda}{\lambda+\mu} f(s)-\frac{\lambda}{\lambda+\mu} f(s+\lambda+\mu), \\
& q_{10}(s)=g(s+\lambda), \\
& q_{12}(s)=g(s)-g(s+\lambda), \\
& q_{21}(s)=\frac{\mu}{\lambda+\mu} f(s)-\frac{\mu}{\lambda+\mu} f(s+\lambda+\mu), \\
& q_{23}(s)=\frac{\lambda}{\lambda+\mu} f(s)+\frac{\mu}{\lambda+\mu} f(s+\lambda+\mu), \\
& q_{32}(s)=g(s),
\end{aligned}
$$

where

$$
f(s) \equiv \int_{0}^{\infty} \mathrm{e}^{-s t} \mathrm{~d} F(t)
$$

and

$$
g(s) \equiv \int_{0}^{\infty} \mathrm{e}^{-s t} \mathrm{~d} G(t)
$$

In general, the small letter functions denote the $L S$ transforms of the corresponding capital ones throughout this paper. It is evident that

$$
\sum_{j=0}^{3} q_{i j}(\infty)=1 \quad(i=0,1,2,3) .
$$

\section{DISTRIBUTION OF THE DISAPPOINTMENT TIME}

Using the mass functions $Q_{i j}(t)$, we derive the distribution of the disappointment time, given that the process starts in state 0 at time 0 . Let $\Phi_{i}(t)$ denote the distribution of the disappointement time starting in state $i$ at time 0 .

Consider that the process starts in state 1. Then the disappointment time occurs in the following two : (i) A need occurs while the unit is under repair. (ii) The process makes a transition into state 0 and after that the process obeys $\Phi_{0}(t)$. These two events are mutually exclusive. Thus we have 


$$
\Phi_{1}(t)=\int_{0}^{t} G(u) \lambda \mathrm{e}^{-\lambda u} \mathrm{~d} u+\int_{0}^{t} \Phi_{0}(t-u) \mathrm{d} Q_{10}(u),
$$

where $G(t) \equiv 1-G(t)$. If the process starts in state 0 , then we have

$$
\Phi_{0}(t)=Q_{03}(t)+\int_{0}^{t} \Phi_{1}(t-u) \mathrm{d} Q_{01}(u) .
$$

Taking the $L S$ transforms on both sides in (20) and (21) and solving with respect to $\varphi_{0}(s)$, we have

where

$$
\varphi_{0}(s)=\frac{q_{03}(s)+q_{01}(s) \mathrm{d}(s)}{1-q_{01}(s) q_{10}(s)},
$$

$$
d(s) \equiv \int_{0}^{\infty} \mathrm{e}^{-s t} G(t) \lambda \mathrm{e}^{-\lambda t} \mathrm{~d} t=\frac{\lambda}{s+\lambda}[1-g(s+\lambda)] .
$$

Substituting (12)-(14) into (22), we have

$$
\begin{aligned}
\varphi_{0}(s)= & \frac{\lambda}{s+\lambda}+ \\
& +\frac{\frac{\lambda}{\lambda+\mu}[f(s)-f(s+\lambda+\mu)]-\frac{\lambda}{\lambda+\mu}\left[1-\frac{\mu}{\lambda+\mu} f(s)-\frac{\lambda}{\lambda+\mu} f(s+\lambda+\mu)\right]}{1-g(s+\lambda)\left[\frac{\mu}{\lambda+\mu} f(s)+\frac{\lambda}{\lambda+\mu} f(s+\lambda+\mu)\right]} .
\end{aligned}
$$

The mean of the disappointment time is given by

$$
T_{0} \equiv \lim _{s \rightarrow 0} \frac{1-\varphi_{0}(s)}{s}=\frac{1}{\lambda}+\frac{\frac{1}{\alpha}-\frac{1}{\alpha+\mu}[1-f(\lambda+\mu)]}{1-g(\lambda)\left[\frac{\mu}{\lambda+\mu}+\frac{\lambda}{\lambda+\mu} f(\lambda+\mu)\right]},
$$

where $\frac{1}{\alpha} \equiv \int_{0}^{\infty} t \mathrm{~d} F(t)$, the mean time to failure of the unit. Note that (24) and (25) are coincident with (17) and (18) in Gaver [4], respectively.

\section{EXPECTED NUMBER OF DISAPPOINTMENTS}

Consider the expected number of the disappointments during the interval $(0, t]$ when the process starts in state 0 at time 0 . Let $M_{i}(t)$ denote the expected number of the disappointment during $(0, t]$ when the process starts in state $i(i=0,1,2)$ at time 0 . Recall that the disappointment occurs either when $\mathrm{n}^{\circ}$ juin $1975, \mathrm{~V}-2$ 
the process makes a transition into state 3 , or when a need occurs while the unit is under repair. Thus we have

$$
\begin{aligned}
M_{1}(t)=\bar{G}(t)\left(1-\mathrm{e}^{-\lambda t}\right)+\int_{0}^{t} M_{0}(t-u) \mathrm{d} Q_{10}(u)+ \\
+\int_{0}^{t}\left[1+M_{2}(t-u)\right] \mathrm{d} Q_{12}(u) .
\end{aligned}
$$

The first term of the right-hand side represents the probability that the disappoint time has occurred up to time $t$ because of occurrence of a need, and the second and third terms represent the expected numbers of disappointments after the process makes transitions into states 0 and 2 (this causes one disappointment), respectively. In a similar fashion,

$$
\begin{aligned}
M_{2}(t)=\int_{0}^{t} \bar{G}(t-u) \mathrm{d} Q_{23}(u)+ & \int_{0}^{t} M_{1}(t-u) \mathrm{d} Q_{21}(u)+ \\
& +\int_{0}^{t}\left[1+M_{2}(t-u)\right] \mathrm{d}\left[Q_{23} * Q_{32}(u)\right], \\
M_{0}(t)=\int_{0}^{t} \bar{G}(t-u) \mathrm{d} Q_{03}(u)+ & \int_{0}^{t} M_{1}(t-u) \mathrm{d} Q_{01}(u)+ \\
& +\int_{0}^{t}\left[1+M_{2}(t-u)\right] \mathrm{d}\left[Q_{03} * Q_{32}(u)\right],
\end{aligned}
$$

where an asterisk denotes the Stieltjes convolution, i.e.

$$
a * b(t) \equiv \int_{0}^{t} b(t-u) \mathrm{d} a(u)
$$

for any $a(t)$ and $b(t)$.

Taking the $L S$ transforms in (26) and (27), and substituting them into (28), we have

$$
\begin{aligned}
m_{0}(s)=\left[q_{01}(s) q_{12}(s)\right. & q_{23}(s)+q_{03}(s)\left[1-q_{12}(s) q_{21}(s)\right]+ \\
& \left.+d(s)\left\{q_{01}(s)\left[1-q_{23}(s) q_{32}(s)\right]+q_{03}(s) q_{32}(s) q_{21}(s)\right\}\right] \\
& /\left\{\left[1-q_{01}(s) q_{10}(s)\right]\left[1-q_{23}(s) q_{32}(s)\right]\right. \\
& \left.-q_{21}(s)\left[q_{12}(s)+q_{10}(s) q_{03}(s) q_{32}(s)\right]\right\}
\end{aligned}
$$


or

$$
\begin{aligned}
& m_{0}(s)=\left\{\frac{\lambda}{\lambda+\mu}[f(s)-f(s+\lambda+\mu)]+[g(s)-g(s+\lambda)] f(s) f(s+\lambda+\mu)+\right. \\
& \left.+\frac{\lambda}{s+\lambda}[1-g(s+\lambda)]\left[\frac{\mu}{\lambda+\mu} f(s)+\frac{\lambda}{\lambda+\mu} g(s+\lambda+\mu)-g(s) f(s) f(s+\lambda+\mu)\right]\right\} \\
& /\{[1-f(s) g(s)][1-g(s+\lambda) f(s+\lambda+\mu)]\} .
\end{aligned}
$$

The expected number of disappointments per unit of time in the steadystate exists and is independent of an initial state since both the mean failure and repair times are finite. That is,

$$
\begin{aligned}
M & \equiv \lim _{t \rightarrow \infty} \frac{M_{0}(t)}{t} \\
& =\frac{1-g(\lambda)\left[\frac{\mu}{\lambda+\mu}+\frac{\lambda}{\lambda+\mu} f(\lambda+\mu)\right]}{\left(\frac{1}{\alpha}+\frac{1}{\beta}\right)[1-g(\lambda) f(\lambda+\mu)]} .
\end{aligned}
$$

\section{AVAILABILITY}

Consider the probability that the process is under disappointment at time $t$, i.e., the unit fails (and is under repair) during a usage period, or a need occurs during a unit repair period. Let $P_{i}(t)$ denote the probability that the process is under disappointment at time $t$, given that the process started in state $i(i=0,1,2)$ at time 0 . The probability $P_{0}(t)$ is the so-called pointwise unavailability and

$$
\bar{P}_{0}(t)\left(\equiv 1-P_{0}(t)\right)
$$

the pointwise availability if the process starts in state 0 at time 0 . Further the probability

$$
P \equiv \lim _{t \rightarrow \infty} \frac{1}{t} \int_{0}^{t} P_{0}(u) \mathrm{d} u
$$

is the so-called limiting interval (or steady-state) unavailability and $\bar{P}(\equiv 1-P)$ the limiting interval availability (see Barlow and Proschan $[2$, p. 5]).

By the similar arguments in (26), (27) and (28), we have

$$
\begin{aligned}
P_{0}(t)=\int_{0}^{t} \bar{G}(t-u) \mathrm{d} Q_{03}(u)+\int_{0}^{t} P_{1}(t-u) \mathrm{d} Q_{01}(u)+ \\
\quad+\int_{0}^{t} P_{2}(t-u) \mathrm{d}\left[Q_{03} * Q_{32}(u)\right],
\end{aligned}
$$




$$
\begin{aligned}
& P_{1}(t)=\bar{G}(t)\left(1-\mathrm{e}^{-\lambda t}\right)+\int_{0}^{t} P_{0}(t-u) \mathrm{d} Q_{10}(u)+ \\
& \quad+\int_{0}^{t} P_{2}(t-u) \mathrm{d} Q_{12}(u), \\
& P_{2}(t)=\int_{0}^{t} G(t-u) \mathrm{d} Q_{23}(u)+\int_{0}^{t} P_{1}(t-u) \mathrm{d} Q_{21}(u)+ \\
& \quad+\int_{0}^{t} P_{2}(t-u) \mathrm{d}\left[Q_{23} * Q_{32}(u)\right] .
\end{aligned}
$$
have

Taking the $L S$ transforms in (32), (33) and (34), and arranging them, we

$$
\begin{aligned}
p_{0}(s)=\left\{\left[q_{01}(s)\right.\right. & {\left.\left[1-q_{23}(s) q_{32}(s)\right]+q_{03}(s) q_{32}(s) q_{21}(s)\right] k(s)+} \\
& +\left[q_{01}(s) q_{12}(s) q_{23}(s)+q_{03}(s)\left[1-q_{12}(s) q_{21}(s)\right][1-g(s)]\right\} \\
& /\left\{\left[1-q_{01}(s) q_{10}(s)\right]\left[1-q_{23}(s) q_{32}(s)\right]\right. \\
& \left.-q_{21}(s)\left[q_{12}(s)+q_{10}(s) q_{03}(s) q_{32}(s)\right]\right\}
\end{aligned}
$$

where

$$
k(s) \equiv \int_{0}^{\infty} \mathrm{e}^{-s t} \mathrm{~d}\left[\bar{G}(t)\left(1-\mathrm{e}^{-\lambda t}\right)\right] .
$$

From (12)-(18), we have

$$
\begin{aligned}
p_{0}(s)=\frac{[1}{1-g(s)] f(s)} & \\
& +\left\{\frac{\lambda}{\lambda+\mu}[1-g(s)][1-f(s)]-\frac{s}{\lambda+s}[1-g(s+\lambda)]\right\} \\
& \times\left[\frac{\mu}{\lambda+\mu} f(s)+\frac{\lambda}{\lambda+\mu} f(s+\lambda+\mu)-g(s) f(s) f(s+\lambda+\mu)\right] \\
& /\{[1-f(s) g(s)][1-g(s+\lambda) f(s+\lambda+\mu)]\} .
\end{aligned}
$$

The limiting probability

$$
P \equiv \lim _{t \rightarrow \infty} \frac{1}{t} \int_{0}^{t} P_{0}(t) \mathrm{d} t
$$

exists and independent of the initial state 0 since both the mean failure and repair times are finite.

Then we have

$$
P=\frac{\frac{1}{\beta}}{\frac{1}{\alpha}+\frac{1}{\beta}}-\frac{\frac{1-g(\lambda)}{\lambda} \cdot \frac{\mu}{\lambda+\mu}[1-f(\lambda+\mu)]}{\frac{1}{\alpha}+\frac{1}{\beta}[1-g(\lambda) f(\lambda+\mu)]},
$$

Revue Française d' Automatique, Informatique et Recherche Opérationnelle 
and

$$
\bar{P}=\frac{\frac{1}{\alpha}}{\frac{1}{\alpha}+\frac{1}{\beta}}+\frac{\frac{1-g(\lambda)}{\lambda} \cdot \frac{\mu}{\lambda+\mu}[1-f(\lambda+\mu)]}{\left(\frac{1}{\alpha}+\frac{1}{\beta}\right)[1-g(\lambda) f(\lambda+\mu)]}
$$

which is the steady-state availability. It can be seen that the first term of the right-hand side in (39) is the steady-state availability of the unit and the second term represents the gain of the availability caused by that the unit is used intermittently.

\section{EXAMPLES}

Consider the two examples that both the time to failure and the repair time are distributed exponentially, and that the time to failure is distributed exponentially and the repair time is exponential or constant.

\section{EXAMPLE 1}

$F(t) \equiv 1-\exp (-\alpha t)$ and $G(t) \equiv 1-\exp (-\beta t)$. Then the mean of the disappointment time is

$$
T_{0}=\frac{1}{\lambda}+\frac{(\lambda+\mu)(\lambda+\beta)}{\lambda \alpha(\lambda+\mu+\alpha+\beta)}
$$

which is derived from (25). The expected number of disappointments per unit of time in the steady-state is given by

$$
M=\frac{\lambda \alpha \beta(\lambda+\mu+\alpha+\beta)}{(\alpha+\beta)\left[\lambda^{2}+\lambda(\mu+\alpha+\beta)+\beta\right]}
$$

which comes from (31). From (39) the steady-state availability is

$$
\bar{P}=\frac{\beta}{\alpha+\beta}+\frac{\mu \alpha \beta}{(\alpha+\beta)\left[\lambda^{2}+\lambda(\mu+\alpha+\beta)+\mu \beta\right]} .
$$

\section{EXAMPLE 2}

$$
F(t) \equiv 1-\exp (-\alpha t) \text { and } G(t)=0 \text { for } t<\theta \text { and } G(t)=1 \text { for } t \geqslant \theta \text {. }
$$
Then we have

$$
\begin{aligned}
T_{0} & =\frac{1}{\lambda}+\frac{\lambda+\mu}{\alpha\left[\lambda+(\mu+\alpha)\left(1-\mathrm{e}^{-\lambda \theta}\right)\right]} \\
M & =\frac{\alpha\left[\lambda+(\mu+\alpha)\left(1-\mathrm{e}^{-\lambda \theta}\right)\right]}{(1+\alpha \theta)\left[\lambda+\mu+\alpha\left(1-\mathrm{e}^{-\lambda \theta}\right)\right]} \\
\bar{P} & =\frac{1}{1+\alpha \theta}+\frac{\mu \alpha\left(1-\mathrm{e}^{-\lambda \theta}\right)}{\lambda(1+\alpha \theta)\left[\lambda+\mu\left(1-\mathrm{e}^{-\lambda \theta}\right)\right]} .
\end{aligned}
$$

$\mathrm{n}^{0}$ juin $1975, \mathrm{~V}-2$ 


\section{CONCLUDING REMARKS}

We have successfully obtained the stochastic behavior of an intermittently used system under the assumption that the behavior of the needs obeys an ordinary alternating renewal process with both exponential distributions.

We conclude with a similar model under the assumption that the behavior of the needs obeys an equilibrium alternating renewal process (see Cox [3, p. 85]). In this case we have

and

$$
H_{00}(t)=H_{10}(t) \equiv \frac{\mu}{\lambda+\mu},
$$

$$
H_{01}(t)=H_{11}(t) \equiv \frac{\lambda}{\lambda+\mu},
$$

which are independent of time $t$. Thus the $L S$ transforms of $Q_{i j}(t)$ are given by

$$
\begin{aligned}
& q_{01}(s)=q_{21}(s)=\frac{\mu}{\lambda+\mu} f(s), \\
& q_{03}(s)=q_{23}(s)=\frac{\lambda}{\lambda+\mu} f(s),
\end{aligned}
$$

and $q_{10}(s), q_{12}(s)$, and $q_{32}(s)$ are equal to (14), (15) and (18), respectively.

From (24) the $L S$ transform of the disappointment time distribution is

$$
\varphi_{0}(s)=\frac{\lambda}{\lambda+\mu} f(s)+\frac{1+\frac{\mu}{s+\lambda}[1-g(s+\lambda)]}{1-\frac{. \mu}{\lambda+\mu} f(s) g(s+\lambda)},
$$

and from (25) the mean disappointment time is

$$
T_{0}=\frac{1}{\lambda}+\frac{\frac{1}{\alpha}-\frac{1}{\lambda+\mu}}{1-\frac{\mu}{\lambda+\mu} g(\lambda)} .
$$

From (29) the $L S$ transform of the expected number of disappointments during $(0, t]$ is

$$
m_{0}(s)=\frac{\lambda f(s)}{\lambda+\mu}-\frac{1+\frac{\mu}{s+\lambda}[1-g(s+\lambda)]}{1-f(s) g(s)}
$$

and the expected number of disappointments per unit of time in the steadystate is 


$$
M=\frac{1-\frac{\mu}{\lambda+\mu} g(\lambda)}{\left(\frac{1}{\alpha}+\frac{1}{\beta}\right)} .
$$

The $L S$ transform of the pointwise unavailability is

$$
p_{0}(s)=f(s) \frac{1-g(s)-\frac{\mu}{\lambda+\mu} \cdot \frac{s}{s+\lambda}[1-g(s+\lambda)]}{1-f(s) g(s)}
$$

which comes from (35). Also the steady-state unavailability is

$$
P=\frac{\frac{1}{\beta}}{\frac{1}{\alpha}+\frac{1}{\beta}}-\frac{\frac{\mu}{\lambda+\mu}[1-g(\lambda)]}{\lambda\left(\frac{1}{\alpha}+\frac{1}{\beta}\right)} .
$$

It is finally noted that (40) and (41) are still valid if we assume arbitrary distributions of occurrence and holding times of a need. Of course, $\frac{1}{\alpha}$ and $\frac{1}{\beta}$ are the means of occurrence and holding times, respectively, if they exist and are finite (see Cox [3, p. 85]).

As an example of this model, assume that all the distributions are exponential. Then

\section{EXAmple 3}

$F(t) \equiv 1-\exp (-\alpha t)$ and $G(t) \equiv 1-\exp (-\beta t)$. From (44), (46) and (47), we have the inverse $L S$ transforms in the following :

$$
\begin{aligned}
\Phi_{0}(t)= & \frac{\lambda \alpha}{(\lambda+\mu)\left(\gamma_{1}-\gamma_{2}\right)} \times \\
& \times\left[\frac{\gamma_{1}-\lambda-\mu-\beta}{\gamma_{1}}\left(1-\mathrm{e}^{-\gamma_{1} t}\right)-\frac{\gamma_{2}-\lambda-\mu-\beta}{\gamma_{2}}\left(1-\mathrm{e}^{-\gamma_{2} t}\right)\right], \\
M_{0}(t)= & \frac{\lambda \alpha}{\lambda+\mu}\left[\frac{\beta(\lambda+\mu+\beta)}{(\alpha+\beta)(\lambda+\beta)} t+\frac{\alpha(\lambda+\mu-\alpha)}{(\alpha+\beta)^{2}(\lambda-\alpha)}\left(1-\mathrm{e}^{-(\alpha+\beta) t}\right)-\right. \\
& \left.-\frac{\lambda \mu}{(\lambda+\beta)^{2}(\lambda-\alpha)}\left(1-\mathrm{e}^{-(\lambda+\beta) t}\right)\right],
\end{aligned}
$$

and

$$
\begin{aligned}
\left.P_{0}(t)=\frac{\lambda \alpha}{(\lambda+\mu)(\lambda}-\alpha\right) & \\
\times & {\left[\frac{\lambda+\mu-\alpha}{\alpha \beta}\left(1-\mathrm{e}^{-(\alpha+\beta) t}\right)-\frac{\mu}{\lambda+\beta}\left(1-\mathrm{e}^{-(\alpha+\beta) t}\right)\right], }
\end{aligned}
$$

$\mathrm{n}^{\circ}$ juin $1975, \mathrm{~V}-2$ 
where $\gamma_{1}$ and $\gamma_{2}$ are the roots of the equation

$$
x^{2}+(\lambda+\alpha+\beta) x+\frac{\lambda \alpha(\lambda+\mu+\beta)}{\lambda+\mu}=0 .
$$

\section{REFERENCES}

[1] R. E. Barlow and L. C. Hunter, Reliability Analysis of a One-Unit System, Opns. Res., vol. 9, 1961, pp. 200:208.

[2] R. E. Barlow and F. Proschan, Mathematical Theory of Reliability, Wiley, New York, 1965.

[3] D. R. Cox, Renewal Theory, Methuen, London, 1962.

[4] D. P. Gaver Jr., A Probability Problem Arising in Reliability and Traffic Studies, Opns. Res., vol. 12, 1964, pp. 534-542.

[5] S. OSAKI, An Intermittently Used System with Preventive Maintenance, J. Opns. Res. Soc. Japan, vol. 15, 1972, pp. 102-111.

[6] R. Pyke, Markov Renewal Processes : Definitions and Preliminary Properties, Ann. Math. Statist., vol. 32, 1961, pp. 1231-1242.

[7] V. S. SRINIVASAN, The Effect of Standby Redundancy in System's Failure with Repair Maintenance, Opns. Res., vol. 14, 1966, pp. 1024-1036. 